\title{
Au-In System Studied with Perturbed Angular Correlation Method
}

\author{
P. Wodniecki, A. Kulińska and B. Wodniecka
}

The Henryk Niewodniczański Institute of Nuclear Physics

Radzikowskiego 152, 31-342 Kraków, Poland

\begin{abstract}
The Au-In system was the subject of our comprehensive study with the perturbed angular correlation technique. Bulk samples of all intermetallic phases shown in the phase diagram were prepared to determine the hyperfine interaction parameters and their temperature dependence. A polymorphic phase transformation in the AuIn phase at about $630 \mathrm{~K}$ and a new metastable modification of $\mathrm{Au}_{7} \mathrm{In}_{3}$ after quenching the sample of 30 at.\% In from temperature above $700 \mathrm{~K}$ were found. In addition, formation of the $\mathrm{Au}-\mathrm{In}$ phases of different stoichiometries at the interfaces of thin $\mathrm{Au} / \mathrm{In}$ films was observed. It turned out that the starting composition of bilayer samples, i.e. the thickness ratio of the $\mathrm{Au}$ and In layers, strongly influenced the stoichiometry of the formed compounds.
\end{abstract}

PACS numbers: $31.30 . \mathrm{Gs}, 68.55 . \mathrm{Nq}, 71.20 . \mathrm{Lp}$

\section{Introduction}

The Au-In binary system exhibits a large number of intermetallic phases [1]. Unfortunately, in spite of many experimental data, some regions of its phase diagram are still not sufficiently examined. Some phases are reported to have different crystallographic structures $\left(\mathrm{Au}_{7} \mathrm{In}\right)$, some are evidenced not in all phase diagrams $\left(\mathrm{Au}_{3} \mathrm{In}_{2}\right)$, and the crystallographic structure of AuIn compound is still not exactly known. It was our aim to measure the electric field gradients (EFG) for ${ }^{111}$ In probes in all $\mathrm{Au}$-In compounds and to determine the number of nonequivalent In sites in the respective lattices. The perturbed angular correlation (PAC) method, due to its huge sensitivity for the changes of microstructure in the nearest neighborhood of the radioactive probes, is a perfect tool to study the polymorphic transformations, even in their very early stages. Thus, measurements in a wide temperature range allow us to observe the structural phase transitions and to determine the homogeneity and temperature region of the phase under study. 
Application of the PAC technique to detect the intermetallic compounds formed in the interface region after annealing at elevated temperatures also requires the knowledge of hyperfine interaction of the PAC probes, present in the lattices of the bulks. The electric field gradients at ${ }^{111} \mathrm{In} /{ }^{111} \mathrm{Cd}$ was measured for bulk $\mathrm{Au}_{x} \operatorname{In}_{(1-x)}$ samples of different stoichiometries [2]. The actual position of probe nuclei was obvious due to the fact that the parent ${ }^{111} \mathrm{In}$ is chemically identical with one of the compound constituents. The measurements were performed for each intermetallic compound shown in the $\mathrm{Au}-\mathrm{In}$ phase diagram [1].

\section{Experimental details}

The $\mathrm{Au}-\mathrm{In}$ bulk samples were prepared by melting under argon atmosphere the appropriate amounts of the constituents. The carrier-free solution of ${ }^{111} \mathrm{In}$ in $0.04 \mathrm{M} \mathrm{HCl}$ was earlier dried-out on the Au-foil in order to dope the investigated specimens with the ${ }^{111} \mathrm{In}$-parent isotope of ${ }^{111} \mathrm{Cd}$. An appropriate annealing in sealed and evacuated quartz tubes ensured the homogeneity of the samples. The PAC measurements were performed at different temperatures from $24 \mathrm{~K}$ to $700 \mathrm{~K}$.

The Au/In bilayer films were deposited onto crystalline silicon wafers at room temperature. The relative thicknesses, measured by a quartz oscillator, were adjusted to obtain the desired composition of the samples. Gold layers, 50-80 nm thick, were covered by indium films with thicknesses of 38-154 nm equivalent to the desirable Au:In atomic ratio (1:2, 1:1, 2:1, 2.5:1, 3:1, 4:1). Some $10^{12}$ of ${ }^{111} \mathrm{In}$ atoms were deposited as a submonolayer at the interface via the evaporation from a thin tungsten wire. The PAC spectra were taken at room temperature before and after subsequent 10 -min annealings.

\section{Results and discussion}

\subsection{The bulk samples}

The hexagonal crystallographic structure of the $\mathrm{Au}_{7} \mathrm{In}$ phase is reported as $\mathrm{DO}_{24}\left(\mathrm{Ni}_{3} \mathrm{Ti}\right.$ )-type [3] or the Nd-type [4]. The PAC spectrum (Fig. 1a) obtained for the $A u_{88.1} \operatorname{In}_{11.9}$ sample was described by a large frequency distribution around $11.3(6) \mathrm{MHz}(\delta=33(3) \%)$. Lack of the well-defined EFG excludes the ordered $\mathrm{Ni}_{3}$ Ti-type of structure, as two unique axially symmetric EFGs should be observed in that case. Thus, for this phase the Nd-type of structure proposed by Hiscocks and Hume-Rothery [4] is more likely.

The $\mathrm{Au}_{3}$ In phase is reported as orthorhombic $\mathrm{DO}_{a}$ structure [5]. This phase undergoes an order-disorder transformation between $572 \mathrm{~K}$ and $612 \mathrm{~K}$ [4]. In $\mathrm{Au}_{75} \mathrm{In}_{25}$ compound one nonaxial EFG was evidenced in the PAC pattern (Fig. 1b). The value of quadrupole frequency linearly decreases with the rise of temperature. An ordered-disordered phase transformation in $\mathrm{Au}_{3} \mathrm{In}$, manifested by the increase 

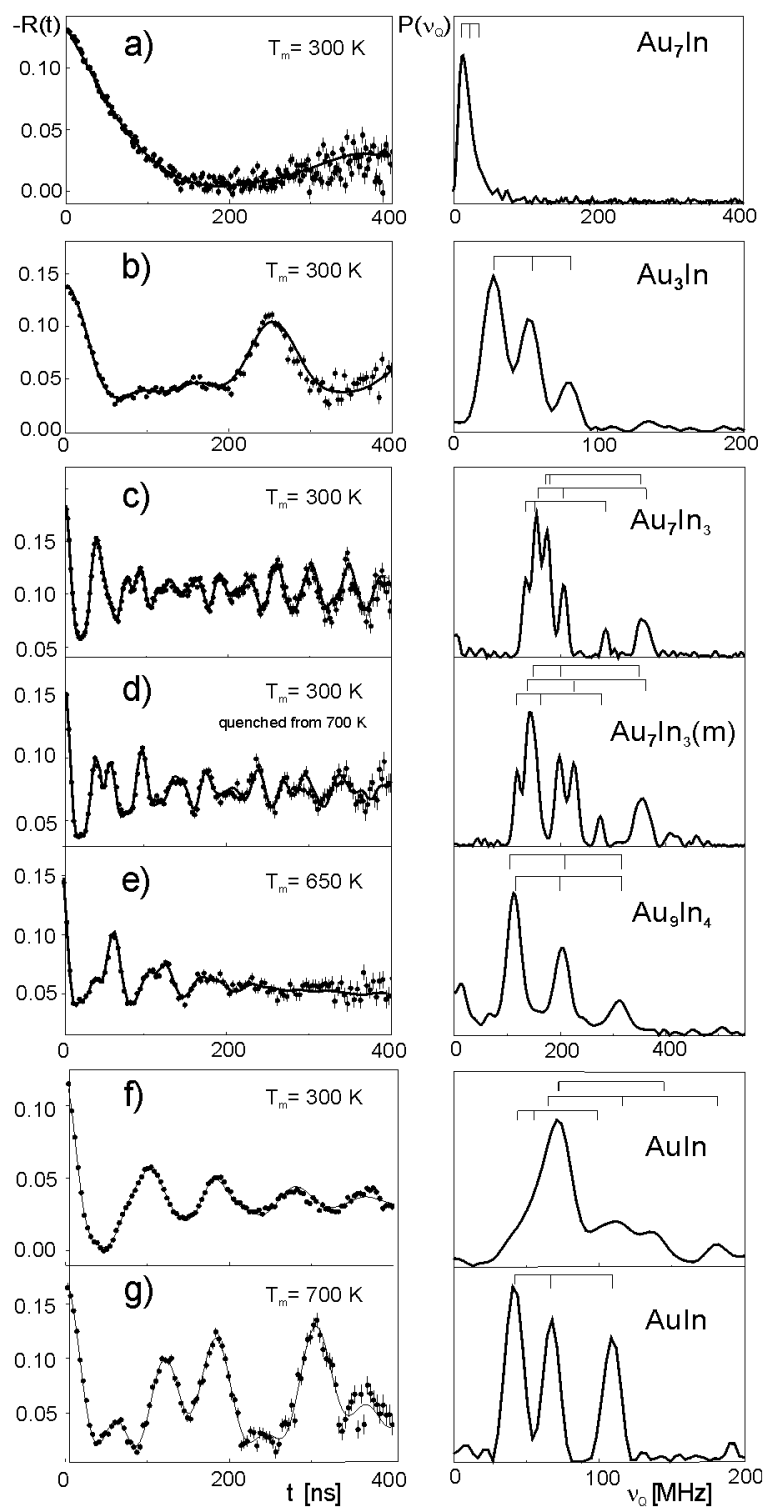

Fig. 1. PAC spectra with the corresponding Fourier transforms for ${ }^{111}$ In doped Au-In bulk samples of different In concentrations taken at indicated temperatures.

in the width $\delta$ of quadrupole frequency distributions above $600 \mathrm{~K}$, was observed in agreement with the phase diagram [1].

For the In concentration of $\sim 30$ at.\% the low- and high-temperature (lt- and ht-) phase are reported. According to the phase diagram at $\sim 650 \mathrm{~K}$ the ht-phase $\left(\mathrm{Au}_{9} \mathrm{In}_{4}\right)$ undergoes a transformation to the lt-phase of $\mathrm{Au}_{7} \mathrm{In}_{3}$ stoichiometry hav- 
ing a different structure. The PAC measurements performed on the $\mathrm{Au}_{70} \mathrm{In}_{30}$ sample below $650 \mathrm{~K}$ resulted in three nonaxial EFGs with equal contributions (Fig. 1c). Above $650 \mathrm{~K}$ two EFGs attributed to indium sites in the $\mathrm{Au}_{9} \mathrm{In}_{4}$ phase (Fig. 1e) were detected. The observed EFG distributions were large, as for the other $\gamma$-brasses [6]. The same sample quenched in water from the temperatures above $700 \mathrm{~K}$ exhibited three new EFGs (see Fig. 1d) which indicates the formation of a new phase. This phase is metastable and undergoes a transformation to the low-temperature phase above $350 \mathrm{~K}$.

The crystallographic structure of the AuIn phase is reported to be triclinic [7], but its structure type is not known. The PAC measurements were performed at different temperatures between $300 \mathrm{~K}$ and $700 \mathrm{~K}$. A change of PAC pattern observed at about $630 \mathrm{~K}$ exhibits a polymorphic phase transformation (Fig. 1f-g). Above this temperature one well-defined quadrupole frequency of $34.7(4) \mathrm{MHz}$ with asymmetry parameter $\eta=0.47(1)$ was measured. Below $630 \mathrm{~K}$ three quadrupole frequencies were necessary to fit the PAC spectra. The detailed PAC experiments proved that this transformation is reproducible and fully reversible. This finding disagrees with the $\mathrm{Au}-\mathrm{In}$ phase diagram [1], where only a single AuIn phase in the whole temperature range of $300-783 \mathrm{~K}$ is reported. To confirm the existence of this phase transition the differential scanning calorimeter measurements were performed with three different scanning rates for heating and cooling the sample (Fig. 2). The temperature, in which the phase transition takes place, determined by this method $(631.8(4) \mathrm{K}$ ) agrees very well with the value obtained via PAC.
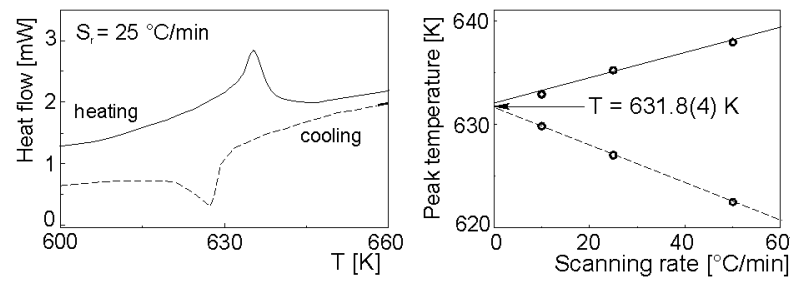

Fig. 2. DSC results obtained for AuIn compound; left: measurements performed for the scanning rate $25^{\circ} \mathrm{C} / \mathrm{min}$; right: results obtained for three different scanning rates used during heating and cooling process.

The results for $\mathrm{Au}_{62} \mathrm{In}_{38}$ stoichiometry did not confirm the existence of the ordered phase reported by some authors [7]. Also an attempt to observe the $\beta_{1}$ phase of 22 at.\% indium concentration with $\mathrm{Cu}_{10} \mathrm{Sb}_{3}$ type of structure [8], where only unique nonaxially symmetric EFG is expected, failed.

In the set of experiments for $\mathrm{Au} / \mathrm{In}$ bilayers having ${ }^{111} \mathrm{In}$ probes deposited at the interfaces the temperature-induced formation of different $\mathrm{Au}$-In phases was evidenced [9]. A fast interdiffusion of the component metals caused bypassing of some phases and the stoichiometry of the "final" compound (i.e. after the final 

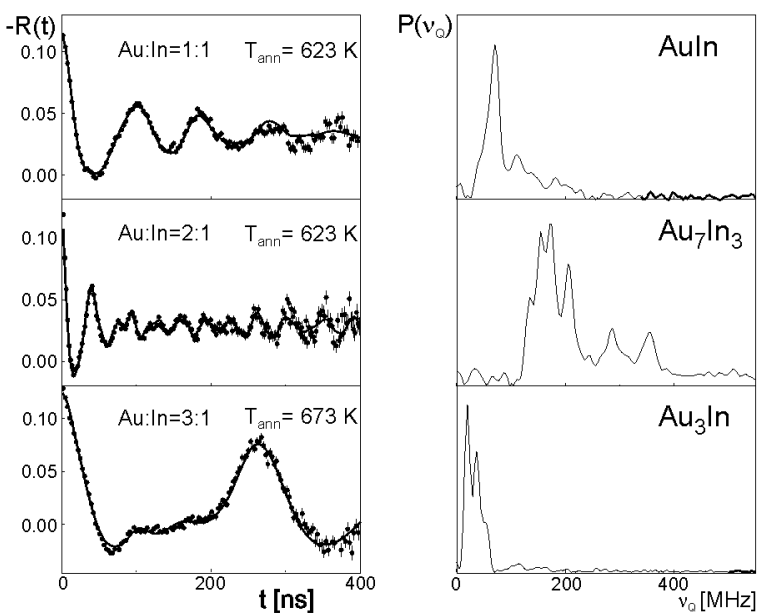

Fig. 3. Room temperature PAC spectra (with corresponding Fourier transforms) of ${ }^{111} \mathrm{Cd}$ in three $\mathrm{Au} / \mathrm{In}$ bilayer samples taken after the final annealing at indicated temperatures.

annealing) formed in the bilayer, was governed by the starting Au:In atomic ratio. Figure 3 shows the PAC spectra for three $\mathrm{Au} / \mathrm{In}$ bilayers of different atomic compositions, taken after formation of the corresponding stoichiometric phases.

The investigations presented here prove that the PAC method is very suitable for a detail study of the phase diagrams and of the phase formation at the interfaces of thin films.

\section{Acknowledgments}

The authors would like to express their appreciation to Prof. J. Mayer for executing the DSC measurements for AuIn phase. This work was supported in part by the State Committee for Scientific Research (grant No. 2P03B 066 18).

\section{References}

[1] Binary Alloys Phase Diagrams, Ed. T.B. Massalski, ASM, Material Park, Ohio 1990 , p. 381.

[2] P. Wodniecki, A. Kulińska, B. Wodniecka, A.Z. Hrynkiewicz, Z. Naturforsch. A 53, 349 (1998).

[3] J. Wegst, K. Schubert, Z. Metallkd. 49, 533 (1958).

[4] S.E.R. Hiscocks, W. Hume-Rothery, Proc. R. Soc. Lond. A 282, 318 (1964).

[5] Pearson's Handbook of Crystallographic Data, Eds. P. Villars, L.D. Calved, 1991, p. 1301

[6] P. Wodniecki, B. Wodniecka, M. Marszałek, A.Z. Hrynkiewicz, Acta Phys. Pol. A 88, 333 (1995). 
[7] K. Schubert, U. Rösler, L. Härle, Naturwissenschaften 40, 437 (1953).

[8] K. Schubert, M. Balk, S. Bhan, H. Breimer, P. Esslinger, E. Stolz, Naturwissenschaften 46, 647 (1959).

[9] A. Kulińska, P. Wodniecki, B. Wodniecka, A.Z. Hrynkiewicz, Hyperfine Interact. 120/121, 319 (1999). 